\title{
3D WAVE FIELD PHASE RETRIEVAL FROM MULTI-PLANE OBSERVATIONS
}

\author{
Artem Migukin, Vladimir Katkovnik, Jaakko Astola \\ Department of Signal Processing, Tampere University of Technology (TUT) \\ Korkeakoulunkatu 10, FI-33720, Tampere, Finland \\ e-mail: artem.migukin@tut.fi,vladimir.katkovnik@tut.fi,jaakko.astola@tut.fi
}

\begin{abstract}
We reconstruct a spatially distributed three-dimensional $(3 D)$ wave field from a number of intensity observations, obtained in different sensor planes, parallel to the object plane. The proposed algorithm can be treated as a multiple plane iterative Gerchberg-Saxton algorithm [1]. It is obtained from the best linear estimate of the complex-valued object distribution derived for the complex-valued observations. This estimator is modified for the intensity measurements in the sensor planes. The algorithm is studied by numerical experiments performed for amplitude and phase object distributions. It is shown that the proposed method allows reconstructing the whole $3 D$ wave fields for different setup parameters. This technique can be applied for $3 D$ imaging. The comparison versus the successive iterative method [2] shows an accuracy advantage of the proposed algorithm provided that the type of modulation in the object plane is known.
\end{abstract}

Index Terms - Imaging, information retrieval, holography, iterative methods, inverse problems, phase estimation

\section{INTRODUCTION}

The reconstruction of the whole wave field (both amplitude and phase) is an important problem utilized in different technical and scientific applications, in particular for $3 D$ imaging or nondestructive testing. The phase can not be measured directly, thus we recover the phase from a number of intensity measurements. There are two groups of the wave field reconstruction methods: interferometric one with a reference beam and methods without a reference beam (phase retrieval). The phase retrieval techniques are much more reliable and technically simpler than the interferometric ones, in particular, because of the simplicity of the optical setup. Furthermore, the phase retrieval approach is more robust with respect to various disturbances (e.g. vibrations).

This research is supported by the Academy of Finland, project No. 213462 (Finnish Centre of Excellence program 2006 - 2011), and the post graduate work of Artem Migukin is funded by the Tampere Graduate School in Information Science and Engineering (TISE).
However, mathematically and computationally the phase retrieval from the magnitude measurements is not a trivial problem. In this paper we study a novel technique based on the parallel usage of the observations from all sensor planes simultaneously for the reconstruction of the $2 D$ wave field in the object plane and $3 D$ wave field distributions in the observation planes. The proposed technique is very different from the established successive methods where the missing phase data are reconstructed by modeling wave field propagation successively from one plane to the next following one.

The planar laser beam scattered by an object propagates through the space. The intensity of the resulting wave field distribution is registered by digital sensors in the sensor planes parallel to the object plane (see Fig.1).

The Gerchberg-Saxton-Fienup iterative algorithm is the most popular phase recovery method, initially proposed for a single measurement plane. It is based on the essential usage of a prior knowledge on the object size and object type (phase or amplitude modulation of the wave field) [1], [3]. The further development and generalization of this technique results in various modifications for different applications (e.g. [4], [5]). For instance, in [6] a multi-plane modification of the algorithm is developed in order to obtain a desired wave field distributions in different planes.

In this work we consider the wave field distribution in the object plane as the only unknown of the problem which one-to-one defines the wave fields for sensor planes. In this approach prior information on the object such as the size and modulation type is used in order to improve the accuracy of the wave field reconstruction. The main contribution of this paper concerns the development of the $3 D$ wave field phase retrieval algorithm and numerical comparative analysis of the proposed parallel phase retrieval algorithm versus the successive method presented in [2]. The influence of the prior information in the object plane on the wave field reconstruction accuracy is analyzed. In the reconstruction algorithms the wave field propagation is performed using the conventional angular spectrum decomposition $(A S D)$. 


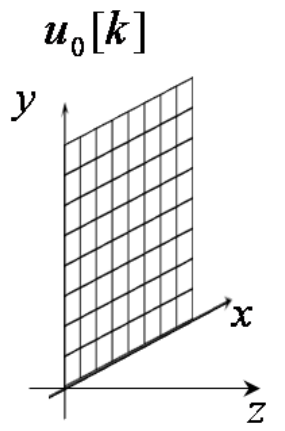

$z=0$

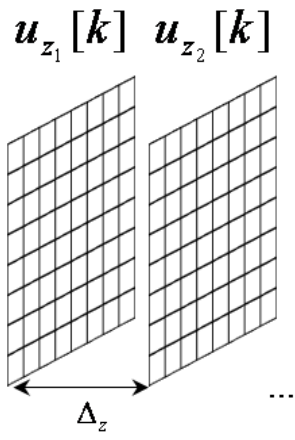

$z_{1} \quad z_{2}$
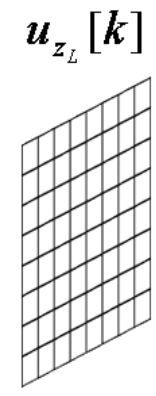

$z_{L}$
Figure 1. Multiple plane wave field reconstruction scenario: $u_{0}[k]$ and $u_{z_{l}}[k]$ are discrete complex amplitudes in the object and measurement planes respectively, $l=1, \ldots, L$.

\section{WAVE FIELD PROPAGATION MODEL}

Let $u_{0}(x)$ and $u_{z_{l}}(x), x \in \mathbb{R}^{2}, l=1, \ldots, L$, denote the complex-valued wave field distributions in the object and sensor planes, respectively. $z_{l}=z_{1}+(l-1) \cdot \Delta_{z}$ indicates a distance between the parallel object and $l-t h$ sensor planes, $\Delta_{z}$ is a distance between two sensor planes, $z_{1}$ is a distance from the object to the first measurement plane and $L$ is a number of the observation planes (sensor positions). We assume that the wave field distributions in the object and sensor planes are pixel-wise invariant. Because of this pixelation we obtain the sampled version of the continuous wave field distributions: $u_{0}(x) \rightarrow u_{0}[k]$, $u_{z_{l}}(x) \rightarrow u_{z_{l}}[k]$, where $k=\left(k_{x}, k_{y}\right) \in \mathbb{Z}^{2}$ is a two dimensional vector with integer components. In Fig. 1 this multi-plane phase retrieval model is presented.

Using the $A S D$ modeling the link between $u_{0}[k]=$ $\left|u_{0}[k]\right| \cdot \exp \left(j \cdot \phi_{0}[k]\right)$ and $u_{z_{l}}[k]=\left|u_{z_{l}}[k]\right| \cdot \exp \left(j \cdot \phi_{z_{l}}[k]\right)$ is given in the frequency domain as

$$
U_{z_{l}}[f]=A S D_{z_{l}, z_{o}}[f] \cdot U_{o}[f] .
$$

Here $f=\left(f_{x}, f_{y}\right) \in \mathbb{Z}^{2}$ is the spatial frequency, $U_{z_{l}}[f]$ and $U_{o}[f]$ are calculated as the $2 D$ Fourier transform of $u_{o}[k]$ and $u_{z_{l}}[k]$ using $\mathcal{F} \mathcal{F} \mathcal{T}$ and the $A S D$ discrete transfer function is given analytically as [8]:

$$
A S D_{z_{l}, z_{o}}[f]=\exp \left(j 2 \pi z_{l} / \lambda \cdot \sqrt{1-\frac{\lambda^{2}}{N_{l}^{2} \cdot \Delta^{2}}\|f\|^{2}}\right),
$$

where $\|f\|_{2}$ is the Euclidean norm, $\|f\|^{2}<\left(N_{l}^{2} \cdot \Delta^{2}\right) /\left(\lambda^{2}\right)$, $\lambda$ is the wavelength, $\Delta$ is the pixel size (we assume that the pixels are square $\Delta \times \Delta$ ) and $N_{l} \times N_{l}$ is the size (in pixels) of the wave field distribution in the $l-t h$ observation plane.

\section{PHASE RETRIEVAL ALGORITHM}

Let us assume for a while that in the sensor planes the complex-valued observations are available. Then the best least square estimate of $U_{o}[f]$ from the observed $U_{z_{l}}[f]$ is calculated from the following optimization problem

$$
\hat{U}_{o}[f]=\arg \min _{U_{o}[f]} \sum_{l=1}^{L}\left\|U_{z_{l}}[f]-A S D_{z_{l}, z_{o}}[f] \cdot U_{o}[f]\right\|_{2}^{2} .
$$

It can be shown that the optimal estimate of $\hat{U}_{o}[f]$ is of the form

$$
\hat{U}_{o}[f]=\frac{1}{L} \sum_{l=1}^{L} A S D_{z_{l}, z_{o}}^{*}[f] \cdot U_{z_{l}}[f],
$$

where ' $*$ ' stands for a complex-conjugate variable.

Let us apply the solution (4) for the considered scenario, when only the intensities of the complex-valued distributions are measured.

Assume that the observations are defined as

$$
o_{z_{l}}[k]=\sqrt{\left|u_{z_{l}}[k]\right|^{2}+\varepsilon_{l}[k]},
$$

where $\varepsilon_{l}[k] \sim \mathcal{N}\left(0, \sigma^{2}\right)$ is the Gaussian noise.

The proposed phase retrieval algorithm is recursive and defined for $A S D$ by the equations:

$$
\begin{aligned}
& \hat{Q}_{0, l}^{(t)}[f]=\frac{A S D_{z_{l}, z_{o}}^{*}[f]}{L} \cdot \mathcal{F} \mathcal{F} \mathcal{T}\left\{v_{l}^{(t-1)}[k]\right\} \\
& \hat{q}_{0, l}^{(t)}[k]=\mathcal{F} \mathcal{F} \mathcal{T}^{-1}\left\{\hat{Q}_{0, l}^{(t)}[f]\right\}, \hat{u}_{0}^{(t)}[k]=\sum_{l=1}^{L} \hat{q}_{0, l}^{(t)}[k], \\
& \hat{u}_{z_{l}}^{(t)}[k]=\mathcal{F} \mathcal{F} \mathcal{T}^{-1}\left\{A S D_{z_{l}, z_{o}}[f] \cdot \mathcal{F} \mathcal{F} \mathcal{T}\left\{\hat{u}_{0}^{(t)}[k]\right\}\right\}, \\
& \hat{\phi}_{z_{l}}^{(t)}[k]=\operatorname{angle}\left(\hat{u}_{z_{l}}^{(t)}[k]\right), \\
& v_{l}^{(t)}[k]=o_{z_{l}}[k] \cdot \exp \left(j \cdot \hat{\phi}_{z_{l}}^{(t)}[k]\right), t=1,2 \ldots
\end{aligned}
$$

The expressions (6) define the iterative multiple plane parallel algorithm, which can be treated as a generalization of the Gerchberg-Saxton algorithm. The first two equations of this algorithm define the object wave field estimate, obtained from estimates in the sensor planes (backward propagation). The complex-valued $\hat{u}_{o}^{(t)}[k]$ can be corrected according to a prior information about the object distribution. The estimates in the sensor planes are exploited in parallel for the calculation of the object estimate. The estimate in the object plane is used for prediction (forward propagation) in the sensor planes (third equation). The intensities of these predictions are corrected by the given intensity observations (the fifth equation in (6)).

In the single-beam multiple-intensity phase reconstruction $(S B M I R)$ algorithm [2] the phase reconstruction is produced by the wave field propagation modeling from one sensor plane to the next following one with a circle loop going from the last sensor plane to the first one. The study of this algorithm demonstrates the efficiency of this technique in simulations and for real experimental data [7]. The proposed technique (6) is essentially different from $S B M I R$ by its structure because the observations from all planes are 

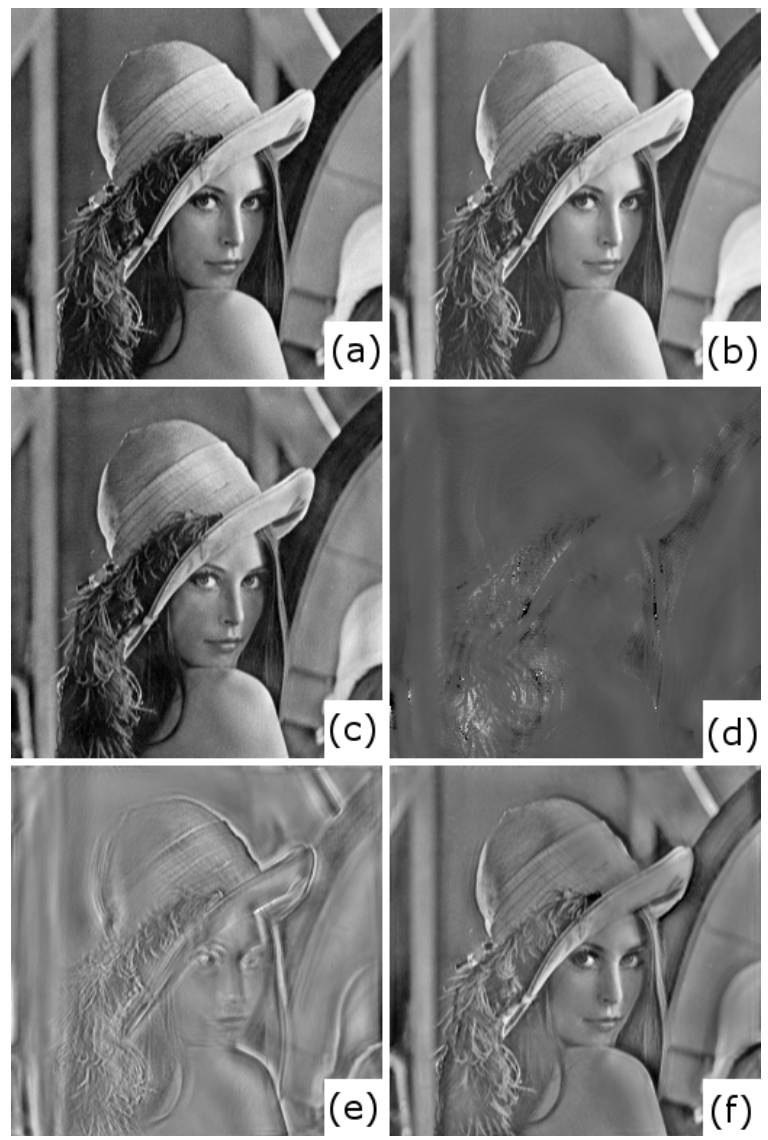

Figure 2. The object wave field reconstruction, $\sigma=0.01, L=10, z_{1}=$ $1.5 \cdot z_{f}$ : (a) $\left|\hat{u}_{0}\right|, A M, R M S E=0.011$, (b) $\hat{\phi}_{0}, P M, R M S E=0.11$, (c) $\left|\hat{u}_{0}\right|, A M, R M S E=0.044$, (d) $\hat{\phi}_{0}, A M, R M S E=0.198$, (e) $\left|\hat{u}_{0}\right|, P M, R M S E=0.147$, (f) $\hat{\phi}_{0}, P M, R M S E=0.175$.

processed in parallel while in $S B M I R$ a plane-to-plane phase reconstruction is used. The use of the object distribution as the only estimated variable enables the parallel algorithm to involve a prior information on the type of the object distribution (the amplitude or phase distribution of $\left.u_{o}[k]\right)$ as well as the object size. This additional information has a significant influence on the reconstruction accuracy. Simulation confirms the advantage of this parallel processing, when the prior information (that the object is of the amplitude or phase type) is used in the algorithm. We assume that the size of the reconstructed object is known.

\section{NUMERICAL EXPERIMENTS}

The numerical experiments are performed for amplitude and phase object distributions with the test-image lena. The images are square $N \times N, N=256$ with the square pixels $\Delta \times \Delta$ of the same size in the object and sensor planes, $\Delta=6.7 \mu \mathrm{m}$, the wavelength $\lambda=632.8 \mathrm{~nm}$. The "infocus" distance is calculated as $z_{f}=N \Delta^{2} / \lambda=18.16$ $\mathrm{mm}$. It is assumed that the additive noise in (5) is zeromean Gaussian with $\sigma=0.01$ and $\sigma=0.05$. The number

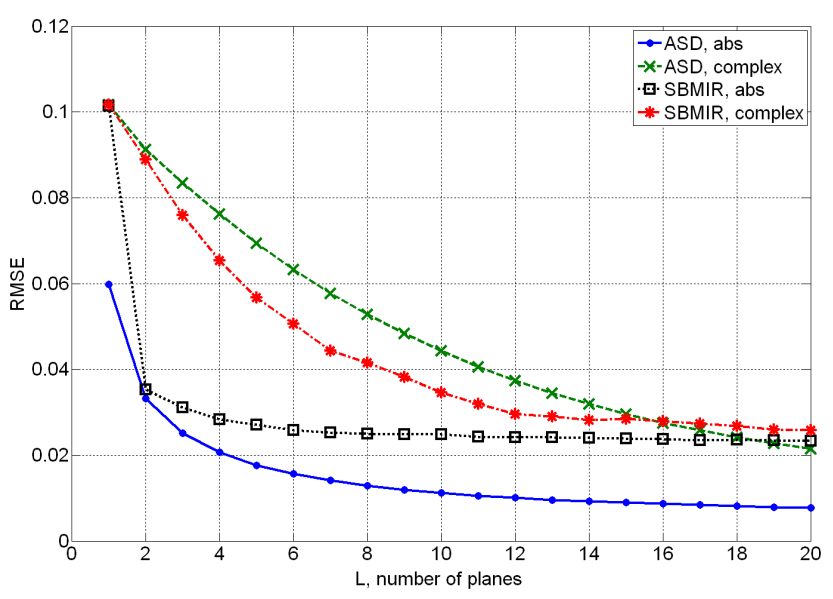

Figure 3. The RMSE reconstruction accuracy of the object magnitude versus the number of planes $L, A M, \sigma=0.01, z_{1}=1.5 \cdot z_{f}$ : the proposed parallel algorithm versus $S B M I R$.

of measurement planes varies: $L=[1,20]$. The results are shown for 100 iterations of the algorithm. The distance between the measurement planes is fixed: $\Delta_{z}=0.5 \mathrm{~mm}$. The influence of the quantization of the observations on the wave field reconstruction accuracy is out of the scope in this work, and we assume that a high precision data from a sensor is given.

Imaging of the reconstructed amplitude $\left|\hat{u}_{0}\right|$ and phase $\hat{\phi}_{0}$ distributions in the object plane is shown in Fig.2. These images correspond to the amplitude $(A M)$ and phase modulation $(P M)$ of the object distribution. We demonstrate the influence of the knowledge of the type modulation priori on the quality of imaging. Here we show the reconstruction for the amplitude (Fig.2 (a)) and phase (Fig.2 (b)) object distributions provided that it is known in advance the corresponding object modulation. If the type of the distribution is unknown in advance, both the amplitude and phase are estimated in the object plane. We show the amplitude and phase estimates for the $A M$ in Fig.2 (c) and (d), and for $P M$ in Fig.2 (e) and (f) respectively. Here and further the wave field reconstruction accuracy is given via the root mean square error $(R M S E)$.

In Fig. 3 we compare the object wave field reconstruction accuracy (for $A M$ ), obtained by the proposed algorithm (6) and by the successive $S B M I R$. The original successive iterative process of $S B M I R$ has no direct connection to the object plane, and it is not able to use the prior information on its distribution (see "SBMIR, complex"). We have modified this algorithm and included the object plane in this successive recursive procedure. The corresponding result is shown as "SBMIR,abs". The curves in Fig. 3 show that the proposed algorithm gives a better accuracy for the amplitude object, when the type of the object distribution is used for the estimation (" $A S D, a b s "$ ). If we do not use the prior information on the object distribution and estimate the object distribution as a complex- 


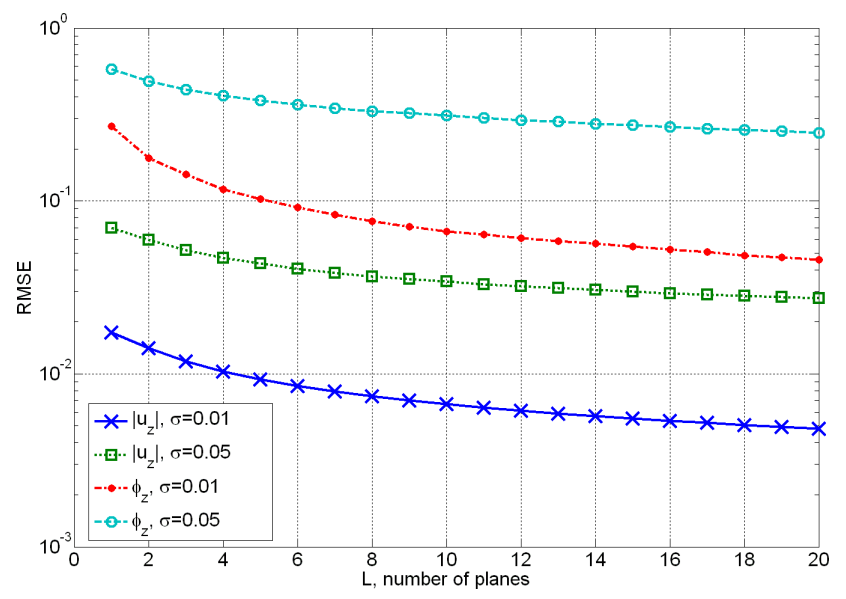

Figure 4. The reconstruction accuracy of the complex-valued wave field (the amplitude $\left|u_{z}\right|$ and phase $\phi_{z}$ ) in the sensor planes, obtained using the proposed algorithm for different noise levels $\sigma, A M$ : the mean values of RMSE for $L$ planes, $z_{1}=1.5 \cdot z_{f}$.

valued one, the $S B M I R$ algorithm gives a better accuracy than the proposed algorithm. In this case the result for the parallel algorithm is marked as "ASD, complex". The $S B M I R$ algorithm converges very quickly and the increase of the number of iterations does not yield to a significant improvement in accuracy. The accuracy value for the proposed method increases monotonically, but slower than for $S B M I R$. For large number of iterations and $L$ the proposed algorithm demonstrates better accuracy.

In Fig.4 the quality of the wave field reconstruction in the sensor planes (for the proposed method) versus the number of the observation planes $L$ is illustrated. The accuracy of $3 D$ wave field for all measurement planes is considered with calculation of $R M S E$ for the phase and amplitude separately. We present the mean value of $R M S E$ over all measurement planes, because these values for different planes are quite close with the standard deviation from the mean values not more than $6 \%$. It is seen that if $\sigma$ is larger, the improvement of wave field reconstruction is not so essential for larger $L$ (a slope of the RMSE curves decreases).

It is found that a larger number of the observation planes $L$ results in monotonically better accuracy for both the object and sensor planes. This improvement is valuable for small $L$ (say, $L=2,3$ ) and not essential for larger $L$. For instance (for $A M$ ), two additional planes from $L=3$ to $L=5$ improves the accuracy in $R M S E$ values by approximately $40 \%$ for the object wave field reconstruction and approximately $20 \%$ for the wave field reconstruction in the sensor planes. $L=10$ results in the further improvement: more than $90 \%$ and $40 \%$ in comparison with $L=3$ for the object and sensor planes respectively.

In the reconstruction of the whole complex-valued object wave field the concordance of the phase estimates $\hat{\phi}_{z_{l}}[k]$ is of very importance, because the phase component of the final object estimate (the sum in the second equation of the algorithm (6)) should have close values. The wrapping effect could lead to a quite strong damage for the parallel algorithm, poor quality of reconstruction and imaging of the final estimates. In Fig.2 (d) an example of this wrapping effect for $A M$ can be seen. The phase wrapping effects in the reconstructed phase distributions are seen as bright white sports. Note that it also results in the worse reconstruction of the object amplitude.

\section{CONCLUSIONS}

In this work we present a phase retrieval algorithm based on simultaneous processing of the data, obtained in a number of parallel observation planes. Numerical experiments demonstrate the applicability of the proposed algorithm for the reconstruction of the complex-valued wave field distributions. The improvement of the reconstruction accuracy depending on the increase of the number of observation planes is shown. The prior information on the object type allows the proposed algorithm to yield better accuracy with respect to the successive $S B M I R$ algorithm.

\section{REFERENCES}

[1] R. W. Gerchberg and W. O. Saxton, "A practical algorithm for the determination of phase from image and diffraction plane pictures," Optik, vol. 35, pp. 227-246, 1972.

[2] G. Pedrini, W. Osten, and Y. Zhang, "Wave-front reconstruction from a sequence of interferograms recorded at different planes," Opt. Lett. vol. 30, pp. 833-835, 2005.

[3] J. R. Fienup, "Reconstruction of an object from the modulus of its Fourier transform," Optics Lett., vol. 3, pp. 27-29, 1978.

[4] G. Yang, et. al., "Gerchberg-Saxton and Yang-Gu algorithms for phase retrieval in a nonunitary transform system: a comparison," Appl. Opt. vol. 33, pp. 209-218, 1994.

[5] R. Piestun and J. Shamir, "Synthesis of 3D light fields and applications," Proc. of the IEEE, vol. 90, pp. 222244, 2002.

[6] G. Sinclair, et al., "Interactive application in holographic optical tweezers of a multi-plane GerchbergSaxton algorithm for three-dimensional light shaping," Opt. Express, vol.12, pp. 1665-1670, 2004.

[7] P. Almoro, G. Pedrini and W. Osten, "Complete wavefront reconstruction using sequential intensity measurements of a volume speckle field," Appl. Opt., vol. 45, pp. 8596-8605, 2006.

[8] J. W. Goodman, Introduction to Fourier Optics, New York: McGraw-Hill Inc, Second Edition, 1996 